\title{
Nonlinear photogalvanic effect induced by monopolar spin orientation of holes in QWs
}

\author{
S.D. Ganichev ${ }^{\mathrm{a}, \mathrm{b}}$, *, S.N. Danilov ${ }^{\mathrm{b}}$, V.V. Bel'kovc, E.L. Ivchenko ${ }^{\mathrm{c}}$, H. Ketterl $^{\mathrm{a}}$, \\ L.E. Vorobjev ${ }^{\mathrm{b}}$, M. Bichler ${ }^{\mathrm{d}}$, W. Wegscheider ${ }^{\mathrm{a}}$, W. Prettl ${ }^{\mathrm{a}}$ \\ anstitut für Experimentelle und Angewandte Physik, Universität Regensburg, 93040 Regensburg, Germany \\ ${ }^{\mathrm{b}}$ St. Petersburg State Technical University, 195251 St. Petersburg, Russia \\ ${ }^{\mathrm{c}}$ A.F. Ioffe Physico-Technical Institute, Russian Academy of Sciences 194021 St. Petersburg, Russia \\ ${ }^{\mathrm{d}}$ Walter Schottky Institut, TU München, 85748 Garching, Germany
}

\begin{abstract}
We report on the first observation of the circular photogalvanic effect (CPGE) induced by optical monopolar spin orientation of holes in p-doped quantum-well structures. It is shown that monopolar optical spin orientation of free carriers causes an electric current which reverses its direction upon changing from left to right circularly polarized radiation. The effect has been observed in ( $\left.\begin{array}{lll}0 & 0 & 1\end{array}\right)$ - and ( 311 ) A-oriented p-GaAs/AlGaAs quantum wells at normal incidence of far-infrared radiation. The photocurrent is proportional to the light intensity at low power levels and gradually saturates with increasing intensity. The CPGE can be utilized to investigate separately spin polarization of electrons and holes and to determine spin-relaxation times. (c) 2001 Elsevier Science B.V. All rights reserved.
\end{abstract}

PACS: 73.50.Mx; 73.50.Pz; 68.85.tq; 78.30.Fs

Keywords: Photogalvanic effect; Monopolar spin orientation

\section{Introduction}

Spin polarization and spin relaxation in semiconductors are the subject of intensive studies of

\footnotetext{
* Corresponding author. Institut für Experimentelle und Angewandte Physik, Universität Regensburg, 93040 Regensburg, Germany. Tel.: +49-941-943-2050; fax: +49-941-943-4223.

E-mail address: sergey.ganichev@physik.uni-regensburg.de (S.D. Ganichev).
}

spin-polarized electron transport aimed at the development of spintronic devices like a spin transistor Refs. [1,2]. So far, optical spin polarization has been achieved by interband optical absorption of circularly polarized light with the photogeneration of spin-polarized electrons and holes Refs. [3-5]. Here we report on the first observation of the circular photogalvanic effect (CPGE) under intersubband transitions in quantum-well $(\mathrm{QW})$ structures. By observing 
the CPGE we experimentally demonstrate monopolar optical spin orientation as a result of absorption of intense terahertz radiation. At normal incidence the current occurs in the plane of the quantum well. The current reverses its direction upon changing the handedness of light circular polarization from right to left. Phenomenologically, the effect is interpreted as a transfer of angular momenta of circularly polarized photons to the directed movement of free carriers, electrons or holes, and therefore depends on the symmetry of the medium. Microscopically, it arises due to optical spin orientation of holes in QWs and asymmetric spin-dependent scattering of spin-polarized carriers followed by an appearance of an electric current. The two states of circular polarization $\sigma_{ \pm}$result in different spin orientations and, thus, in electric photocurrents of opposite directions. In contrast to the case of interband optical excitation, under intersubband transitions only one kind of carriers is involved leading to a monopolar spin orientation. The CPGE saturates at high radiation intensities. The saturation is controlled by both spin relaxation and energy relaxation of free carriers which allows the investigation of spin relaxation processes in QWs.

\section{Experimental technique and samples}

The experiments have been carried out on (0 0 1)-MOCVD grown p-GaAs/AlGaAs multi QW structures with 300 or 400 undoped wells of $20 \mathrm{~nm}$ width separated by $10 \mathrm{~nm}$ wide doped barriers and on a p-GaAs/AlGaAs (3 1 1)A-MBE grown single QW and $20 \mathrm{QWs}$ width of $15 \mathrm{~nm}$. The surface of the $(001)$-grown $\mathrm{QW}$ was tilted by an angle of $5^{\circ}$ between the crystallographic [001] direction and the sample surface normal as has been verified by $\mathrm{X}$-ray diffraction. Samples of $5 \times 5 \mathrm{~mm}^{2}$ size with hole densities $p_{\mathrm{s}}$ in the QWs varying from $10^{11} \mathrm{~cm}^{-2}$ to $2 \times 10^{12} \mathrm{~cm}^{-2}$ have been investigated. Two pairs of ohmic contacts (see inset in Fig. 1) have been prepared along [1 $1 \overline{1} 0]$ (direction 1-2) and [1 10 ] for (0 0 1)-grown GaAs/AlGaAs and [1 $1 \overline{1} 0$ ] (direction 1-2) and [2 33 3] for (3 11 1)A-grown GaAs/AlGaAs, respectively.

A high power far-infrared molecular laser pumped by a TEA- $\mathrm{CO}_{2}$ laser has been used as a radiation source delivering $100 \mathrm{~ns}$ pulses with the intensity $I$

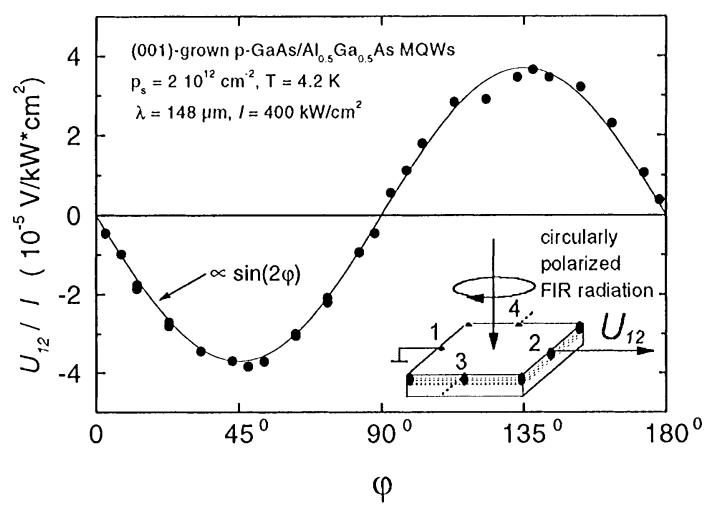

Fig. 1. CPGE voltage signal $U_{12}$ picked up across the contact pair $1 \& 2$ and normalized by the intensity $I$ as a function of the phase angle $\varphi$. The full line is fitted by using Eq. (3) $\left(U_{12} \propto \sin 2 \varphi\right)$. Inset shows geometry of experiment.

up to $1 \mathrm{MW} / \mathrm{cm}^{2} . \mathrm{NH}_{3}$ has been used as a FIR laser medium yielding strong linearly polarized emissions at wavelengths $\lambda=35,76,90$, and $148 \mu \mathrm{m}$ Refs. [6,7]. The photon energies of the laser lines correspond to transitions between various heavy-hole and/or light-hole subbands of p-GaAs QWs Ref. [8]. The linearly polarized laser light could be modified to circularly polarized radiation by applying crystalline quartz $\lambda / 4$-plates.

\section{Circular and linear photogalvanic effect}

Irradiating the GaAs QWs by normally incident circularly polarized radiation (see inset in Fig. 1) we have observed a fast e.m.f. signal $U_{12}$ in unbiased samples across one contact pair (contacts $1 \& 2$ in the inset). The photocurrent changes sign if the circular polarization is switched from $\sigma_{+}$to $\sigma_{-}$. Measurements of $U_{12}$ as a function of the angle $\varphi$ between the optical axis of the $\lambda / 4$-plate and the plane of polarization of the laser radiation, reveal that the photogalvanic current $\boldsymbol{j}$ is proportional to the degree of circular polarization $P_{\text {circ }}=\sin 2 \varphi$ (Fig. 1). The magnitude and the sign of the CPGE are practically unchanged with variation of the angle of incidence in the range from $-40^{\circ}$ to $+40^{\circ}$. The photogalvanic signal picked up at the other pair of contacts (contacts $3 \& 4$ in the inset of Fig. 1) does not change sign by switching the circular 
polarization from $\sigma_{+}$to $\sigma_{-}$. The signal $U_{34}$ is periodic in $\varphi$ with the period being one half of that for $U_{12}$.

The photogalvanic current under study can be described by the following phenomenological expression

$j_{\lambda}=\chi_{\lambda \mu v}\left(E_{\mu} E_{v}^{*}+E_{v} E_{\mu}^{*}\right) / 2+\gamma_{\lambda \mu} i\left(\boldsymbol{E} \times \boldsymbol{E}^{*}\right)_{\mu}$,

where $\boldsymbol{E}$ is the complex amplitude of the electric field of the electromagnetic wave and

$i\left(\boldsymbol{E} \times \boldsymbol{E}^{*}\right)_{\mu}=P_{\text {circ }} E_{0}^{2} \frac{\boldsymbol{q}}{q} \propto \sin 2 \varphi$

$E_{0}=|\boldsymbol{E}|, \boldsymbol{q}$ is the light wavevector. In a bulk crystal, $\lambda=x, y, z$, while in a QW structure grown along the $z$ direction, say $z$ parallel [ $\left[\begin{array}{ll}0 & 0\end{array}\right]$, the index $\lambda$ runs only over the in-plane axes $x, y$ because the barriers prevent carriers from moving along the $z$ axis and, definitely, $j_{z}=0$. For the QW structure grown along $\mathrm{z} \|[\mathrm{h}, \mathrm{h}, 1]$, we choose $x$ and $y$ to be parallel to the axis $[1, \overline{1}, 0]$ and $[1,1,(\overline{2 h})]$, respectively. The photocurrent given by the tensor $\chi$ describes the so-called linear photogalvanic effect (LPGE) because it is usually observed under linearly polarized optical excitation. The circular photogalvanic effect (CPGE) is described by the pseudotensor $\gamma$ and can be observed only under circularly polarized excitation, $P_{\text {circ }} \neq 0$. It is defined as a generation of photocurrent which changes its polarity under a change of sign of the circular polarization of the exciting light. The CPGE is allowed in systems having a point group symmetry where at least some components of a polar and an axial vector transform according to identical representation. In the particular case of CPGE, a polar vector is the current density $\boldsymbol{j}$ and an axial vector is the vector product $\boldsymbol{e} \times \boldsymbol{e}^{*}$ with $\boldsymbol{e}$ being the complex light polarization unit vector. Note, that for a transverse wave one has $i\left(\boldsymbol{e} \times \boldsymbol{e}^{*}\right)=P_{\text {circ }} \boldsymbol{q} / q$, where $\boldsymbol{q}$ is the light wavevector and $P_{\text {circ }}$ is the degree of circular polarization. The same symmetry allows linear terms in the electron wavevector $\boldsymbol{k}$ in the effective Hamiltonian $H^{(1)}=\beta_{l m \sigma l} k_{m}$, where $\sigma_{l}$ are the Pauli matrices.

The point group $\mathrm{C}_{\mathrm{s}}$ of the investigated (3 11 )Aand tilted (001)-grown samples contains only two elements: the identity and one mirror reflection. In this case, under normal incidence and for the experiment directions $x$ (contacts 1\&2) and $y$ (contacts 3\&4), we have for circularly polarized radiation

$j_{x}=E_{0}^{2} \gamma \sin 2 \varphi, \quad j_{y}=E_{0}^{2}\left(\chi_{+}+\chi_{-} \cos ^{2} 2 \varphi\right)$,

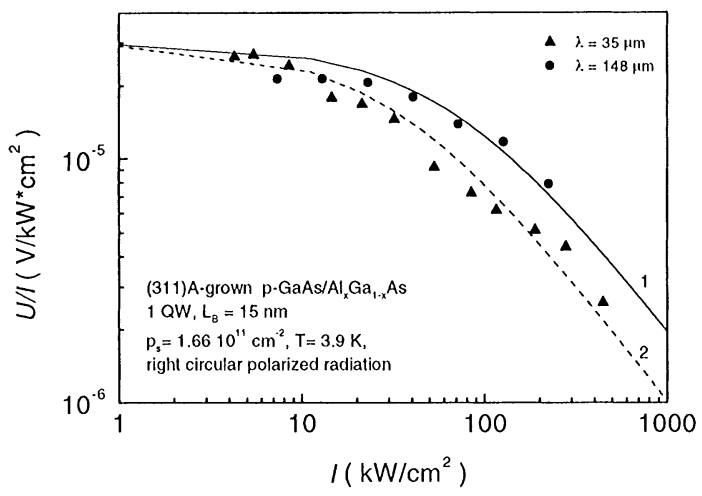

Fig. 2. CPGE voltage signal $U_{12}$ normalized by the intensity $I$ as a function of $I$ for two different wavelengths. Lines are fitted by using Eq. (5) with the saturation intensities $I_{\mathrm{S}}=70 \mathrm{~kW} / \mathrm{cm}^{2}$ (1) and $I_{\mathrm{s}}=35 \mathrm{~kW} / \mathrm{cm}^{2}(2)$.

and linearly polarized radiation

$j_{x}=E_{0}^{2} \chi_{1} \sin 2 \alpha, \quad j_{y}=E_{0}^{2}\left(\chi_{+}+\chi_{-} \cos 2 \alpha\right)$,

where $\alpha$ is the angle between the electric field vector of linearly polarized radiation and the axis $x, \gamma=\gamma_{x z}, \chi_{1}=$ $\chi_{x x y}$ and $\chi_{ \pm}=\left(\chi_{y x x} \pm \chi_{y y y}\right) / 2$. The left side relation in (3) describes the circular photocurrent whereas the right equation in (3) and the both equations in (4) give the current due to the LPGE. Experimentally the angular dependencies of photocurrents given by Eqs. (3) and (4) have been observed in good agreement to the theoretical relations.

\section{Saturation of photogalvanic effect}

The intensity dependence of CPGE and LPGE has been measured in the range of $1-10^{3} \mathrm{~kW} / \mathrm{cm}^{2}$ for all wavelengths revealing a saturation with increasing intensity. This saturation is observable in a wide range of temperatures and hole densities. The photocurrent depends on the radiation intensity $I$ as

$j \propto \frac{I}{1+I / I_{\mathrm{S}}}$,

where $I_{\mathrm{s}}$ is the saturation intensity (see for CPGE Fig. 2). The magnitude $I_{\mathrm{s}}$ for CPGE is in general smaller than for LPGE, varies with the radiation wavelength and increases with increasing temperature (Fig. 3) and doping density. At room temperature the 


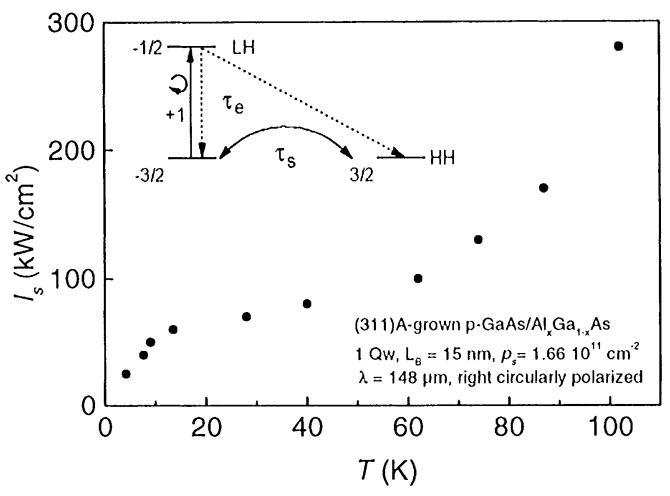

Fig. 3. Temperature dependence of the saturation intensity $I_{\mathrm{S}}$ of the CPGE. Inset: schematic representation of one-photon intersubband transitions and relaxation (dashed lines) under the excitation by $\sigma_{+}$right circularly polarized photons.

saturation intensity is higher than $1 \mathrm{MW} / \mathrm{cm}^{2}$ and cannot be determined with the available technique.

Saturation of one-photon absorption of circular polarized light may be caused by spin orientation of the hole gas. For any subband $v$, at nonzero in-plane wavevector $\boldsymbol{k}$, each of the two degenerate hole states $|v k j\rangle$ is an admixture of all four angular momentum components $J_{z}= \pm \frac{3}{2}, \pm \frac{1}{2}$, yet with different weights so that the quantum-mechanical average $\left\langle J_{z}\right\rangle$ does not vanish. For circularly polarized radiation the rates for direct one-photon intersubband transitions $|v \boldsymbol{k} j\rangle \rightarrow$ $\left|v^{\prime} \boldsymbol{k} j^{\prime}\right\rangle$ depend on the indices $j, j^{\prime}$ resulting in a selective population of the $j$ and $j^{\prime}$ states, corresponding to a monopolar spin orientation. Direct intersubband transitions by circularly polarized light depopulate and populate selectively spin states at certain energies in the valence band due to selection rules and energy and momentum conservation (see inset in Fig. 3). Since the transition rate is proportional to the difference of populations in the initial and final states, the increasing selective population tends to equalize the differing transition rates and, hence, leads to a sublinear dependence of the CPGE on the intensity. The saturation is controlled either by the hole spin relaxation or by energy relaxation of photoexcited carriers. In contrast to circularly polarized light, optical transitions induced by the linearly polarized light are not spin selective and the saturation is controlled by energy relaxation of photoexcited carriers only. Therefore comparison between the saturation of CPGE and LPGE may allow to determine the spin relaxation time.

In general, two mechanisms contribute to CPGE, photoexcitation and scattering of photoexcited carriers. The first contribution, $j_{1}$, is caused by the asymmetry of the momentum distribution of carriers excited in the optical transitions which are sensitive to the light circular polarization due to selection rules Ref. [9]. The second contribution, $j_{2}$, is a result of spin relaxation of optically oriented carriers (see Ref. [10] and references therein). The currents $j_{1}$ and $j_{2}$ can be estimated as $j_{1,2} \approx e w s_{0} \beta$, where $e$ is the elementary charge, $w$ is the probability of photon absorption per unit time and unit volume in the case of multiple quantum wells (or per unit area for a single quantum well), $s_{0}$ is the average spin of photoexcited carriers at the moment of generation, and $\beta$ is one of the coefficients $\beta_{l m}$ in the $\boldsymbol{k}$-linear Hamiltonian $H^{(1)}$. Although $j_{1}$ and $j_{2}$ can be of the same order of magnitude, the physical difference between the two contributions becomes obvious after the light is switched of: $j_{1}$ decays with the carrier momentum relaxation time $\tau_{p}$ whereas the decay of $j_{2}$ is governed by the spin relaxation time $\tau_{s}$ which can be much longer than $\tau_{p}$.

The saturation of the photogalvanic current can be due to spin relaxation for CPGE and slow energy relaxation in the case of both CPGE and LPGE.

For linearly polarized radiation the photon absorption rate $w$ is proportional to the incident light intensity $I$ at low intensities and saturates as

$w=A \frac{I}{1+I / I_{\mathrm{se}}}$,

with one saturation intensity $I_{\text {se }}$ determined by the energy relaxation time $\tau_{e} ; A$ is a constant. Circularly polarized excitation results in the orientation of spins which, in turn, leads to an additional mechanism of saturation. This can be described by adding to $w$ a spin-dependent contribution and writing the probability rate as $w(1-\eta s)$, where $s$ is the spin polarization in the nonequlibrium steady state and $\eta$ is a constant. From the balance between generation and relaxation of spin polarization we obtain

$s=\frac{1}{\eta} \frac{I}{I_{\mathrm{sp}}} \frac{1}{1+I\left(I_{\mathrm{se}}^{-1}+I_{\mathrm{sp}}^{-1}\right)}$,

where $I_{\mathrm{sp}}=p_{\mathrm{s}}\left(s_{0} A \eta \tau_{\mathrm{s}}\right)^{-1}$. Thus the parameter $I_{\mathrm{S}}$ in Eq. (5) is equal to $I_{\mathrm{se}}$ or $I_{\mathrm{se}} \cdot I_{\mathrm{sp}} /\left(I_{\mathrm{se}}+I_{\mathrm{sp}}\right)$ for LPGE or CPGE, respectively. 
The experimentally observed saturation of the CPGE and the LPGE follows Eqs. (6) and (7). Saturation intensities have been measured for a wide range of temperatures, hole densities, and photon energies, corresponding to different initial and final energy levels of holes, varying from $30 \mathrm{~kW} / \mathrm{cm}^{2}$ to non-measurably large values. The estimation of relaxation times needs the knowledge of the photon absorption rate at low intensities which is not yet available but might be calculated after Ref. [8]. The experimentally observed difference between CPGE and LPGE saturation gives evidence for spin controlled relaxation and allows to distinguish between energy and spin relaxation.

\section{Acknowledgements}

Financial support by the DFG, NATO Linkage Grant, INTAS and the RFFI are gratefully acknowledged.

\section{References}

[1] S. Datta, B. Das, Appl. Phys. Lett. 56 (1990) 665.

[2] G. Prinz, Phys. Today 48 (1995) 58.

[3] F. Meier, B.P. Zacharchenia (Eds.), Optical Orientation, Elsevier, Amsterdam, 1984.

[4] J.M. Kikkawa, D.D. Awschalom, Nature 397 (1999) 139.

[5] J.M. Kikkawa, D.D. Awschalom, Phys. Rev. Lett. 80 (1998) 4313.

[6] S.D. Ganichev, W. Prettl, I.N. Yassievich, Rev. in Phys. Solid State 39 (1997) 1703.

[7] S.D. Ganichev, Physica B 273-274 (1999) 737.

[8] L.E. Vorobjev, D.V. Donetskii, L.E. Golub, JETP Lett. 63 (1996) 981.

[9] E.L. Ivchenko, Yu.B. Lyanda-Geller, G.E. Pikus, Sov. Phys. JETP 73 (1990) 550.

[10] E.L. Ivchenko, G.E. Pikus, Sov. Phys. JETP Lett. 27 (1978) 604. 